\title{
An introduction to harmonic analysis on the infinite symmetric group
}

\author{
Grigori Olshanski
}

August 20, 2018

\section{Introduction}

The aim of the present survey paper is to provide an accessible introduction to a new chapter of representation theory - harmonic analysis for noncommutative groups with infinite-dimensional dual space.

I omitted detailed proofs but tried to explain the main ideas of the theory and its connections with other fields. The fact that irreducible representations of the groups in question depend on infinitely many parameters leads to a number of new effects which never occurred in conventional noncommutative harmonic analysis. A link with stochastic point processes is especially emphasized.

The exposition focuses on a single group, the infinite symmetric group $S(\infty)$. The reason is that presently this particular example is worked out the most. Furthermore, $S(\infty)$ can serve as a very good model for more complicated groups like the infinite-dimensional unitary group $U(\infty)$.

The paper is organized as follows. In $\S 1$, I explain what is the problem of harmonic analysis for $S(\infty)$. $\S \S 2-5$ contain the necessary preparatory material. In $\S 6$, the main result is stated. It was obtained in a cycle of papers by Alexei Borodin and myself. In $\S 7$, the scheme of the proof is outlined. The final $\S 8$ contains additional comments and detailed references.

This paper is an expanded version of lectures I gave at the Euler Institute, St.-Petersburg, during the NATO ASI Program "Asymptotic combinatorics with applications to mathematical physics". I also partly used the material of my lectures at the Weizmann Institute of Science, Rehovot. I am grateful to Anatoly Vershik, Amitai Regev, and Anthony Joseph for warm hospitality in St. Petersburg and Rehovot, and to Vladimir Berkovich for taking notes of my lectures at the Weizmann. Finally, I would like to thank Alexei Borodin for cooperation and help. 


\section{Virtual permutations and generalized regular representations}

\subsection{The Peter-Weyl theorem}

Let $\mathcal{K}$ be a compact group, $\mu$ be the normalized Haar measure on $\mathcal{K}$ (i.e., $\mu(\mathcal{K})=1)$, and $H$ be the Hilbert space $L^{2}(K, \mu)$. The group $\mathcal{G}=\mathcal{K} \times \mathcal{K}$ acts on $\mathcal{K}$ on the right as follows: if $g=\left(g_{1}, g_{2}\right) \in G$ and $x \in \mathcal{K}$, then $x \cdot g=g_{2}^{-1} x g_{1}$. This action gives rise to a unitary representation $T$ of $\mathcal{G}$ on $H$ :

$$
(T(g) f)(x)=f(x \cdot g), \quad f \in H, \quad g \in G .
$$

It is called the biregular representation. Let $\widehat{\mathcal{K}}$ be the set of equivalence classes of irreducible representations of $\mathcal{K}$. Recall that all of them are finite dimensional and unitarizable. For $\pi \in \widehat{\mathcal{K}}$, let $\bar{\pi}$ denote the dual representation. Since $\pi$ is unitary, $\bar{\pi}$ is obtained from $\pi$ by the conjugation automorphism of the base field $\mathbb{C}$.

Peter-Weyl's Theorem. The biregular representation $T$ is equivalent to the direct sum of the irreducible representations of $G$ of the form $\pi \otimes \bar{\pi}$,

$$
T \sim \bigoplus_{\pi \in \widehat{\mathcal{K}}}(\pi \otimes \bar{\pi})
$$

This is one of the first results of noncommutative harmonic analysis. The aim of noncommutative harmonic analysis can be stated as decomposing natural representations into irreducible ones. The biregular representation can be called a natural representation because it is fabricated from the group itself in a very natural way. The Peter-Weyl theorem serves as a guiding example for more involved theories of noncommutative harmonic analysis.

\subsection{The infinite symmetric group}

Let $S(n)$ be the symmetric group of degree $n$, i.e., the group of permutations of the set $\{1, \ldots, n\}$. By the very definition, $S(n)$ acts on $\{1, \ldots, n\}$. The stabilizer of $n$ is canonically isomorphic to $S(n-1)$, which makes it possible to define, for any $n=2,3, \ldots$, an embedding $S(n-1) \rightarrow S(n)$. Let $S(\infty)$ be the inductive limit of the groups $S(n)$ taken with respect to these embeddings. We call $S(\infty)$ the infinite symmetric group.

Clearly, $S(\infty)$ is a countable, locally finite group. It can be realized as the group of all finite permutations of the set $\{1,2, \ldots\}$.

\subsection{The biregular representation for $S(\infty)$}

The definition of a biregular representation given in $\S 1.1$ evidently makes sense for the group $S(\infty)$. Namely, set $\mathcal{K}=S(\infty), \mathcal{G}=S(\infty) \times S(\infty)$, and take as $\mu$ the counting measure on $S(\infty)$. Then the unitary representation $T$ of the group 
$S(\infty) \times S(\infty)$ in the Hilbert space $L^{2}(S(\infty), \mu)$ is defined by exactly the same formula as in $\S 1.1$.

Proposition. The biregular representation $T$ of the group $S(\infty) \times S(\infty)$ is irreducible.

Sketch of proof. Let $\operatorname{diag}(S(\infty))$ be the image of $S(\infty)$ under the diagonal embedding $S(\infty) \rightarrow S(\infty) \times S(\infty)$. The Dirac function $\delta_{e}$ is a unique (up to a constant factor) $\operatorname{diag}(S(\infty))$-invariant vector in the space of $T$. This follows from the fact that all conjugacy classes in $S(\infty)$, except for $\{e\}$, are infinite. On the other hand, $\delta_{e}$ is a cyclic vector, i.e., it generates under the action of $S(\infty) \times S(\infty)$ a dense subspace in $L^{2}(S(\infty))$. It follows that there is no proper closed $S(\infty) \times S(\infty)$-invariant subspace.

Thus, in the case of the group $S(\infty)$, the naive analog of the biregular representation is of no interest for harmonic analysis. We will explain how to modify the construction in order to get interesting representations.

From now on we are using the notation

$$
G=S(\infty) \times S(\infty), \quad K=\operatorname{diag}(S(\infty)) .
$$

We call $G$ the infinite bisymmetric group.

\subsection{Virtual permutations}

Note that in the construction of $\S 1.1$, the group $\mathcal{K}$ plays two different roles: it is the carrier of a Hilbert space of functions and it acts (by left and right shifts) in this space. The idea is to separate these two roles. As the carrier of a Hilbert space we will use a remarkable compactification $\mathfrak{S}$ of $S(\infty)$. It is not a group but still a $G$-space, which is sufficient for our purposes.

For any $n \geq 2$, we define a projection $p_{n}: S(n) \rightarrow S(n-1)$ as removing the element $n$ from the cycle containing it. That is, given a permutation $\sigma \in S(n)$, if $n$ is fixed under $\sigma$ then $p_{n}(\sigma)=\sigma$, and if $n$ enters a nontrivial cycle $(\cdots \rightarrow$ $i \rightarrow n \rightarrow j \rightarrow \cdots)$ then we simply replace this cycle by $(\cdots \rightarrow i \rightarrow j \rightarrow \cdots)$. We call $p_{n}$ the canonical projection.

Proposition. The canonical projection $p_{n}: S(n) \rightarrow S(n-1)$ commutes with the left and right shifts by the elements of $S(n-1)$. Moreover, for $n \geq 5$ it is the only map $S(n) \rightarrow S(n-1)$ with such a property.

Let $\mathfrak{S}$ be the projective limit of the finite sets $S(n)$ taken with respect to the canonical projections. Any point $x \in \mathfrak{S}$ is a collection $\left(x_{n}\right)_{n \geq 1}$ such that $x_{n} \in S(n)$ and $p_{n}\left(x_{n}\right)=x_{n-1}$. For any $m$, we identify $S(m)$ with the subset of those points $x=\left(x_{n}\right)$ for which $x_{n} \in S(m)$ for all $n \geq m$. This allows us to embed $S(\infty)$ into $\mathfrak{S}$.

We equip $\mathfrak{S}$ with the projective limit topology. In this way we get a totally disconnected compact topological space. We call it the space of virtual permutations. 
The image of $S(\infty)$ is dense in $\mathfrak{S}$. Hence, $\mathfrak{S}$ is a compactification of the discrete space $S(\infty)$.

There exists an action of the group $G$ on the space $\mathfrak{S}$ by homeomorphisms extending the action of $G$ on $S(\infty)$. Such an action is unique.

There are several different realizations of the space $\mathfrak{S}$. One of them looks as follows. Set $I_{n}=\{0, \ldots, n-1\}$. There exists a bijection

$$
\mathfrak{S} \rightarrow I:=I_{1} \times I_{2} \times \ldots, \quad x=\left(x_{1}, x_{2}, \ldots\right) \mapsto\left(i_{1}, i_{2}, \ldots\right)
$$

such that $i_{n}=0$, if $x_{n}(n)=n$, and $i_{n}=j$, if $x_{n}(n)=j<n$. This bijection is a homeomorphism (here we equip the product space $I$ with the product topology). It gives rise, for every $n \geq 1$, to a bijection $S(n) \rightarrow I_{1} \times \cdots \times I_{n}$. In this realization, the canonical projection $p_{n}: S(n) \rightarrow S(n-1)$ turns into the natural projection $I_{1} \times \cdots \times I_{n} \rightarrow I_{1} \times \cdots \times I_{n-1}$.

\subsection{Ewens' measures on $\mathfrak{S}$}

Let $\mu_{1}^{(n)}$ be the normalized Haar measure on $S(n)$. Its pushforward under the canonical projection $p_{n}$ coincides with the measure $\mu_{1}^{(n-1)}$, because $p_{n}$ commutes with the left (and right) shifts by elements of $S(n-1)$. Thus, the measures $\mu_{1}^{(n)}$ are pairwise consistent with respect to the canonical projections. Hence, we can define their projective limit, $\mu_{1}=\lim _{\longleftarrow} \mu_{1}^{(n)}$, which is a probability measure on S.

The measure $\mu_{1}$ is invariant under the action of $G$, and it is the only probability measure on $\mathfrak{S}$ with this property. Thus, viewing $\mathfrak{S}$ as a substitute of the group space, we may view $\mu_{1}$ as a substitute of the normalized Haar measure.

Now we define a one-parameter family of probability measures containing the measure $\mu_{1}$ as a particular case.

For $t \geq 0$, let $\mu_{t}^{(n)}$ be the following measure on $S(n)$ :

$$
\mu_{t}^{(n)}(x)=\frac{t^{[x]-1}(t+1)(t+2) \cdots \cdot(t+n-1)}{,}
$$

where $[x]=[x]_{n}$ is the number of cycles of $x$ in $S(n)$. If $t=1$ then this reduces to above definition of the measure $\mu_{1}^{(n)}$.

Proposition. (i) $\mu_{t}^{(n)}$ is a probability measure on $S(n)$, i.e.,

$$
\sum_{x \in S(n)} t^{[x]}=t(t+1) \cdots(t+n-1) .
$$

(ii) The measures $\mu_{t}^{(n)}$ are pairwise consistent with respect to the canonical projections.

(iii) The pushforward of $\mu_{t}^{(n)}$ under the bijective map $S(n) \rightarrow I_{1} \times \cdots \times I_{n}$ of $\S 1.4$ is the product measure $\nu_{t}^{(1)} \times \cdots \times \nu_{t}^{(n)}$, where, for any $m, \nu_{t}^{(m)}$ is the 
following probability measure on $I_{m}$ :

$$
\nu_{t}^{(m)}(i)= \begin{cases}\frac{t}{t+m-1}, & i=0 \\ \frac{1}{t+m-1}, & i=1, \ldots, m-1 .\end{cases}
$$

Proof. (i) Induction on $n$. Assume that the equality in question holds for $n-1$. Notice that $\left[p_{n}(x)\right]_{n-1}$ is equal to $[x]_{n}$ when $x \notin S(n-1)$, and to $[x]_{n}-1$ when $x \in S(n-1)$. We have

$$
\begin{aligned}
\sum_{x \in S(n)} t^{[x]} & =\sum_{y \in S(n-1)} \sum_{p_{n}(x)=y} t^{[x]}=\sum_{y \in S(n-1)}\left(t \cdot t^{[y]}+(n-1) t^{[y]}\right) \\
& =t \cdot t(t+1) \cdots(t+n-2)+(n-1) t(t+1) \cdots(t+n-2) \\
& =t(t+1) \cdots(t+n-1) .
\end{aligned}
$$

(ii) We have to verify that for every $y \in S(n-1)$

$$
\frac{t^{[y]-1}(t+1) \cdots(t+n-2)}{=} \sum_{p_{n}(x)=y} \frac{t^{[x]-1}(t+1) \cdots(t+n-1)}{.} .
$$

It is precisely what is done in the proof of (i).

(iii) This follows from the fact that, under the bijection $x \mapsto\left(i_{1}, \ldots, i_{n}\right)$ between $S(n)$ and $I_{1} \times \cdots \times I_{n}$, the number of zeros in $\left(i_{1}, \ldots, i_{n}\right)$ equals $[x]$.

The consistency property makes it possible to define, for any $t \geq 0$, a probability measure $\mu_{t}=\lim \mu_{t}^{(n)}$ on $\mathfrak{S}$. This measure is invariant under the diagonal subgroup $K$ but is not $G$-invariant (except the case $t=1$ ). As $t \rightarrow \infty, \mu_{t}$ tends to the Dirac measure at $e \in S(\infty) \subset \mathfrak{S}$. Let us denote this limit measure by $\mu_{\infty}$.

Following S. V. Kerov, we call the measures $\mu_{t}$ the Ewens measures. The next claim gives a characterization of the family $\left\{\mu_{t}\right\}$.

Proposition. The measures $\mu_{t}$, where $0 \leq t \leq \infty$, are exactly those probability measures on $\mathfrak{S}$ that are $K$-invariant and correspond to product measures on $I_{1} \times I_{2} \times \ldots$.

\subsection{Transformation properties of the Ewens measures}

Recall that $[\sigma]_{n}$ denotes the number of cycles of a permutation $\sigma \in S(n)$.

Proposition. (i) For any $x=\left(x_{n}\right) \in \mathfrak{S}$ and $g \in G$, the quantity $\left[x_{n} \cdot g\right]_{n}-\left[x_{n}\right]_{n}$ does not depend on $n$ provided that $n$ is large enough.

(ii) Denote by $c(x, g)$ the stable value of this quantity. The function $c(x, g)$ is an additive cocycle with values in $\mathbb{Z}$, that is,

$$
c(x, g h)=c(x \cdot g, h)+c(x, g), \quad x \in \mathfrak{S}, \quad g, h \in G .
$$


Recall that a measure is called quasi-invariant under a group of transformations if, under the shift by an arbitrary element of the group, the measure is transformed to an equivalent measure.

Proposition. Assume $t \in(0,+\infty)$.

(i) The measure $\mu_{t}$ is quasi-invariant under the action of the group $G$.

(ii) We have

$$
\frac{\mu_{t}(d(x \cdot g))}{\mu_{t}(d x)}=t^{c(x, g)},
$$

where the left-hand side is the Radon-Nikodym derivative.

Note that $c(x, g)=1$ whenever $g \in K$. This agrees with the fact that the measures are $K$-invariant.

\subsection{The representations $T_{z}$}

We start with a general construction of unitary representations related to group actions on measure spaces with cocycles.

Assume we are given a space $\mathcal{S}$ equipped with a Borel structure (i.e., a distinguished sigma-algebra of sets), a discrete group $\mathcal{G}$ acting on $\mathcal{S}$ on the right and preserving the Borel structure, and a Borel measure $\mu$, which is quasiinvariant under $\mathcal{G}$. A complex valued function $\tau(x, g)$ on $\mathcal{S} \times \mathcal{G}$ is called a multiplicative cocycle if

$$
\tau(x, g h)=\tau(x \cdot g, h) \tau(x, g), \quad x \in \mathcal{S}, \quad g, h \in \mathcal{G} .
$$

Next, assume we are given a multiplicative cocycle $\tau(x, g)$ which is a Borel function in $x$ and which satisfies the relation

$$
|\tau(x, g)|^{2}=\frac{\mu(d(x \cdot g))}{\mu(d x)} .
$$

Then these data allow us to define a unitary representation $T=T_{\tau}$ of the group $\mathcal{G}$ acting in the Hilbert space $L^{2}(\mathcal{S}, \mu)$ according to the formula

$$
(T(g) f)(x)=\tau(x, g) f(x \cdot g), \quad f \in L^{2}(\mathcal{S}, \mu), \quad x \in \mathcal{S}, \quad g \in \mathcal{G} .
$$

Let $z \in \mathbb{C}$ be a nonzero complex number. We apply this general construction for the space $\mathcal{S}=\mathfrak{S}$, the group $\mathcal{G}=G$, the measure $\mu=\mu_{t}$ (where $t=|z|^{2}$ ), and the cocycle $\tau(x, g)=z^{c(x, g)}$. All the assumptions above are satisfied, so that we get a unitary representation $T=T_{z}$ of the group $G$.

Using a continuity argument it is possible to extend the definition of the representations $T_{z}$ to the limit values $z=0$ and $z=\infty$ of the parameter $z$. It turns out that the representation $T_{\infty}$ is equivalent to the biregular representation of §1.3. Thus, the family $\left\{T_{z}\right\}$ can be viewed as a deformation of the biregular representation.

We call the $T_{z}$ 's the generalized regular representations. These representations are reducible (with the only exception of $T_{\infty}$ ). Now we can state the main problem that we address in this paper. 
Problem of harmonic analysis on $S(\infty)$. Describe the decomposition of the generalized regular representations $T_{z}$ into irreducibles ones.

\section{Spherical representations and characters}

\subsection{Spherical representations}

By a spherical representation of the pair $(G, K)$ we mean a pair $(T, \xi)$, where $T$ is a unitary representation of $G$ and $\xi$ is a unit vector in the Hilbert space $H(T)$ such that:

(i) $\xi$ is $K$-invariant and

(ii) $\xi$ is cyclic, i.e., the span of the vectors of the form $T(g) \xi$, where $g \in G$, is dense in $H(T)$.

We call $\xi$ the spherical vector. We call two spherical representations $\left(T_{1}, \xi_{1}\right)$ and $\left(T_{2}, \xi_{2}\right)$ equivalent if there exists an isometric isomorphism between their Hilbert spaces which commutes with the action of $G$ and preserves the spherical vectors. Such an isomorphism is unique within multiplication by a scalar. The equivalence $\left(T_{1}, \xi_{1}\right) \sim\left(T_{2}, \xi_{2}\right)$ implies the equivalence $T_{1} \sim T_{2}$ but the converse is not true in general.

The matrix coefficient $(T(g) \xi, \xi)$, where $g \in G$, is called the spherical function. Two spherical representations are equivalent if and only if their spherical functions coincide. $(G, K)$.

We aim to give an independent characterization of spherical functions for

\subsection{Positive definite functions}

Recall that a complex-valued function $f$ on a group $\mathcal{G}$ is called positive definite if:

(i) $f\left(g^{-1}\right)=\overline{f(g)}$ for any $g \in \mathcal{G}$ and

(ii) for any finite collection $g_{1}, \ldots, g_{n}$ of elements of $\mathcal{G}$, the $n \times n$ Hermitian matrix $\left[f\left(g_{j}^{-1} g_{i}\right)\right]$ is nonnegative.

Positive definite functions on $\mathcal{G}$ are exactly diagonal matrix coefficients of unitary representations of $\mathcal{G}$.

Now return to our pair $(G, K)$. The spherical functions for $(G, K)$ can be characterized as the positive definite, $K$-biinvariant functions on $G$, normalized at $e \in G$.

\subsection{Characters}

Recall that the character of an irreducible representation $\pi$ of a compact group $\mathcal{K}$ is the function $g \mapsto \chi^{\pi}(g)=\operatorname{Tr}(\pi(g))$. If $\mathcal{K}$ is noncompact, an irreducible representation $\pi$ of $\mathcal{K}$ is not necessarily finite dimensional, and so the function $g \mapsto \operatorname{Tr}(\pi(g))$ does not make sense in general. But it turns out that in certain 
cases the ratio

$$
\tilde{\chi}^{\pi}(g)=\frac{\chi^{\pi}(g)}{\chi^{\pi}(e)}
$$

does make sense.

Let $\mathcal{K}$ be an arbitrary group. A function on $\mathcal{K}$ is said to be central if it is constant on conjugacy classes. Denote by $\mathcal{X}(K)$ the set of central, positive definite, normalized functions on $\mathcal{K}$ (if $\mathcal{K}$ is a topological group then we additionally require the functions to be continuous). If $\varphi, \psi \in \mathcal{X}(\mathcal{K})$, then for every $t \in[0,1]$ the function $(1-t) \varphi+t \psi$ is also an element of $\mathcal{X}(\mathcal{K})$, i.e., $\mathcal{X}(\mathcal{K})$ is a convex set.

Recall that a point of a convex set is called extreme if it is not contained in the interior of an interval entirely contained in the set. Let $\operatorname{Ex}(\mathcal{X}(\mathcal{K}))$ denote the subset of extreme points of $\mathcal{X}(\mathcal{K})$.

If the group $\mathcal{K}$ is compact then the functions from $\operatorname{Ex}(\mathcal{X}(\mathcal{K}))$ are exactly the normalized irreducible characters $\widetilde{\chi}^{\pi}(g)$, where $\pi \in \widehat{\mathcal{K}}$. As for general elements of $\mathcal{X}(\mathcal{K})$, they are (possibly infinite) convex linear combinations of these functions.

In particular, if $\mathcal{K}$ is finite then $\mathcal{X}(\mathcal{K})$ is a finite-dimensional simplex.

We will call the elements of $\mathcal{X}(\mathcal{K})$ the characters of $\mathcal{K}$. The elements of $\operatorname{Ex}(\mathcal{X}(\mathcal{K}))$ will be called the extreme characters. Notice that this terminology does not agree with the conventional terminology of representation theory. However, in the case of the group $S(\infty)$ this will not lead to a confusion.

\subsection{Correspondence between spherical representations of $(G, K)$ and characters of $S(\infty)$}

There is a natural 1-1 correspondence between spherical functions for $(G, K)$ and characters of $S(\infty)$. Specifically, given a function $f$ on the group $G=$ $S(\infty) \times S(\infty)$, let $\chi$ be the function on $S(\infty)$ obtained by restricting $f$ to the first copy of $S(\infty)$. Then $f \mapsto \chi$ establishes a 1-1 correspondence between $K$-biinvariant functions on $G$ and central functions on $S(\infty)$. Moreover, this correspondence preserves the positive definiteness property. This implies that the equivalence classes of spherical representations of $(G, K)$ are parametrized by the characters of $S(\infty)$.

Proposition. Let $T$ be a unitary representation of $G$ and $H(T)^{K}$ be the subspace of $K$-invariant vectors in the Hilbert space $H(T)$ of $T$.

If $T$ is irreducible then $H(T)^{K}$ has dimension 0 or 1 . Conversely, if the subspace $H(T)^{K}$ has dimension 1 then its cyclic span is an irreducible subrepresentation of $T$.

Corollary. For an irreducible spherical representation of $(G, K)$, the spherical vector $\xi$ is defined uniquely, within a scalar multiple, which does not affect the spherical function.

A spherical function corresponds to an irreducible representation if and only if the corresponding character is extreme. Thus, the (equivalence classes of) irreducible spherical representations of $(G, K)$ are parametrized by extreme characters of $S(\infty)$. 


\subsection{Spectral decomposition}

Proposition. (i) For any character $\psi \in \mathcal{X}(S(\infty))$, there exists a probability measure $P$ on the set $\operatorname{Ex}(\mathcal{X}(S(\infty)))$ of extreme characters such that

$$
\psi(\sigma)=\int_{\chi \in \operatorname{Ex}(\mathcal{X}(S(\infty)))} \chi(\sigma) P(d \chi), \quad \sigma \in S(\infty) .
$$

(ii) Such a measure is unique.

(iii) Conversely, for any probability measure $P$ on the set of extreme characters, the function $\psi$ defined by the above formula is a character of $S(\infty)$.

We call this integral representation the spectral decomposition of a character. The measure $P$ will be called the spectral measure of $\psi$. If $\psi$ is extreme then its spectral measure reduces to the Dirac mass at $\psi$.

Let $(T, \xi)$ be a spherical representation of $(G, K), \psi$ be the corresponding character, and $P$ be its spectral measure. If $\xi$ is replaced by another spherical vector in the same representation then the character $\psi$ is changed, hence the measure $P$ is changed, too. However, $P$ is transformed to an equivalent measure. Thus, the equivalence class of $P$ is an invariant of $T$ as a unitary representation.

The spectral decomposition of $\psi$ determines a decomposition of the representation $T$ into a continual integral of irreducible spherical representations.

\section{Thoma's theorem and spectral decomposition of the representations $T_{z}$ with $z \in \mathbb{Z}$}

\subsection{First example of extreme characters}

Let $\alpha=\left(\alpha_{1} \geq \cdots \geq \alpha_{p} \geq 0\right)$ and $\beta=\left(\beta_{1} \geq \cdots \geq \beta_{q} \geq 0\right)$ be two collections of numbers such that

$$
\sum_{i=1}^{p} \alpha_{i}+\sum_{j=1}^{q} \beta_{j}=1
$$

Here one of the numbers $p, q$ may be zero (then the corresponding collection $\alpha$ or $\beta$ disappears). To these data we will assign an extreme character $\chi^{(\alpha, \beta)}$ of $S(\infty)$, as follows.

Let

$$
p_{k}(\alpha, \beta)=\sum_{i=1}^{p} \alpha_{i}^{k}+(-1)^{k-1} \sum_{j=1}^{q} \beta_{j}^{k} .
$$

Note that

$$
p_{1}(\alpha, \beta) \equiv 1 \text {. }
$$

Given $\sigma \in S(\infty)$, we denote by $m_{k}(\sigma)$ the number of $k$-cycles in $\sigma$. Since $\sigma$ is a finite permutation, we have

$$
m_{1}(\sigma)=\infty, \quad m_{k}(\sigma)<\infty \text { for } k \geq 2, \quad m_{k}(\sigma)=0 \text { for } k \text { large enough. }
$$


In this notation, we set

$$
\chi^{(\alpha, \beta)}(\sigma)=\prod_{k=1}^{\infty}\left(p_{k}(\alpha, \beta)\right)^{m_{k}(\sigma)}=\prod_{k=2}^{\infty}\left(p_{k}(\alpha, \beta)\right)^{m_{k}(\sigma)}, \quad \sigma \in S(\infty),
$$

where we agree that $1^{\infty}=1$ and $0^{0}=1$.

Proposition. Each function $\chi^{(\alpha, \beta)}$ defined by the above formula is an extreme character of $S(\infty)$.

If $p=1$ and $q=0$ (i.e., $\alpha_{1}=1$ and all other parameters disappear) then we get the trivial character, which equals 1 identically. If $p=0$ and $q=1$ then we get the alternate character $\operatorname{sgn}(\sigma)= \pm 1$, where the plus-minus sign is chosen according to the parity of the permutation. More generally, we have

$$
\chi^{(\alpha, \beta)} \cdot \operatorname{sgn}=\chi^{(\beta, \alpha)} .
$$

\subsection{Thoma's set}

Let $\mathbb{R}^{\infty}$ denote the direct product of countably many copies of $\mathbb{R}$. We equip $\mathbb{R}^{\infty}$ with the product topology. Let $\Omega$ be the subset of $\mathbb{R}^{\infty} \times \mathbb{R}^{\infty}$ formed by couples $\alpha \in \mathbb{R}^{\infty}, \beta \in \mathbb{R}^{\infty}$ such that

$$
\alpha=\left(\alpha_{1} \geq \alpha_{2} \geq \cdots \geq 0\right), \quad \beta=\left(\beta_{1} \geq \beta_{2} \geq \cdots \geq 0\right), \quad \sum_{i=1}^{\infty} \alpha_{i}+\sum_{j=1}^{\infty} \beta_{j} \leq 1 .
$$

We call $\Omega$ the Thoma set. We equip it with topology induced from that of the space $\mathbb{R}^{\infty} \times \mathbb{R}^{\infty}$. It is readily seen that $\Omega$ is a compact space.

The couples $(\alpha, \beta)$ that we dealt with in $\S 3.1$ can be viewed as elements of $\Omega$. The subset of such couples (with given $p, q$ ) will be denoted by $\Omega_{p q}$.

Note that each $\Omega_{p q}$ is isomorphic to a simplex of dimension $p+q-1$. As affine coordinates of the simplex one can take the numbers

$$
\alpha_{1}-\alpha_{2}, \ldots, \alpha_{p-1}-\alpha_{p}, \alpha_{p}, \beta_{1}-\beta_{2}, \ldots, \beta_{q-1}-\beta_{q}, \beta_{q}
$$

but we will not use these coordinates.

Proposition. The union of the simplices $\Omega_{p q}$ is dense in $\Omega$.

For instance, the point $(\underline{0}, \underline{0})=(\alpha \equiv 0, \beta \equiv 0) \in \Omega$ can be approximated by points of the simplices $\Omega_{p 0}$ as $p \rightarrow \infty$,

$$
(\underline{0}, \underline{0})=\lim _{p \rightarrow \infty}((\underbrace{1 / p, \ldots, 1 / p}_{p}), \underline{0}) .
$$




\subsection{Description of extreme characters}

Now we extend by continuity the definition of $\S 3.1$. For any $k=2,3, \ldots$ we define the function $p_{k}$ on $\Omega$ as follows. If $\omega=(\alpha, \beta) \in \Omega$ then

$$
p_{k}(\omega)=p_{k}(\alpha, \beta)=\sum_{i=1}^{\infty} \alpha_{i}^{k}+(-1)^{k-1} \sum_{j=1}^{\infty} \beta_{j}^{k} .
$$

Note that $p_{k}$ is a continuous function on $\Omega$. It should be emphasized that the condition $k \geq 2$ is necessary here: the similar expression with $k=1$ (that is, the sum of all coordinates) is not continuous.

Next, for any $\omega=(\alpha, \beta) \in \Omega$ we set

$$
\chi^{(\omega)}(\sigma)=\chi^{(\alpha, \beta)}(\sigma)=\prod_{k=2}^{\infty}\left(p_{k}(\alpha, \beta)\right)^{m_{k}(\sigma)}, \quad \sigma \in S(\infty),
$$

Thoma's theorem. (i) For any $\omega \in \Omega$ the function $\chi^{(\omega)}$ defined above is an extreme character of $S(\infty)$.

(ii) Each extreme character is obtained in this way.

(iii) Different points $\omega \in \Omega$ define different characters.

In particular, the character $\chi^{(\underline{0}, \underline{0})}$ is the delta function at $e \in S(\infty)$. It corresponds to the biregular representation defined in $\$ 1.3$.

Note that the topology of $\Omega$ agrees with the topology of pointwise convergence of characters on $S(\infty)$. This implies, in particular, that the characters of $\S 3.1$ are dense in the whole set of extreme characters with respect to the topology of pointwise convergence.

Corollary. For any character $\psi$ of $S(\infty)$, its spectral measure $P$ can be viewed as a probability measure on the compact space $\Omega$, and the integral representation of $\S 2.5$ can be rewritten in the following form

$$
\psi(\sigma)=\int_{\Omega} \chi^{(\omega)} P(d \omega), \quad \sigma \in S(\infty) .
$$

\subsection{Spectral decomposition for integral values of $z$}

Consider the generalized regular representations $T_{z}$ of the group $G$ introduced in $\S 1.7$.

Theorem. Assume $z$ is an integer, $z=k \in \mathbb{Z}$.

(i) The representation $T_{k}$ possesses $K$-invariant cyclic vectors, i.e., it can be made a spherical representation.

(ii) Let $\xi$ be any such vector, $\psi$ be the corresponding character, and $P$ be its spectral measure on $\Omega$. Then $P$ is supported by the subset

$$
\bigcup_{p, q \geq 0,(p, q) \neq(0,0), p-q=k} \Omega_{p q}
$$

and for any $\Omega_{p q}$ entering this subset, the restriction of $P$ to $\Omega_{p q}$ is equivalent to Lebesgue measure on the simplex $\Omega_{p q}$. 
When $k \neq 0$, the restriction $(p, q) \neq(0,0)$ is redundant because it follows from the condition $p-q=k$.

The condition $p-q=k$ also implies that the spectral measures corresponding to different integral values of the parameter $z$ are mutually singular. This, in turn, implies that the corresponding representations are disjoint, i.e., they do not have equivalent subrepresentations.

\section{The characters $\chi_{z}$}

\subsection{Definition of $\chi_{z}$ and its explicit expression}

Let $T_{z}$ be a generalized regular representation of $G$. Assume first $z \neq 0$. Recall that $T_{z}$ is realized in the Hilbert space $L^{2}\left(\mathfrak{S}, \mu_{t}\right)$, where $t=|z|^{2}$. Let 1 denote the function on $\mathfrak{S}$ identically equal to 1 . It can be viewed as a vector of $L^{2}\left(\mathfrak{S}, \mu_{t}\right)$. Since $\mu_{t}$ is $K$-invariant and the cocycle $z^{c(x, g)}$ entering the construction of $T_{z}$ is trivial on $K$, the vector $\mathbf{1}$ is a $K$-invariant vector. Consider the corresponding matrix coefficient and pass to the corresponding character (see $\S 2.4)$, which we denote by $\chi_{z}$. Thus,

$$
\chi_{z}(\sigma)=\left(T_{z}(\sigma, e) \mathbf{1}, \mathbf{1}\right), \quad \sigma \in S(\infty) .
$$

We aim to give a formula for $\chi_{z}$. To do this we will describe the expansion of $\left.\chi_{z}\right|_{S(n)}$ in irreducible characters of $S(n)$ for any $n$. Recall that the irreducible representations of $S(n)$ are parametrized by Young diagrams with $n$ boxes. Let $\mathbb{Y}_{n}$ be the set of these diagrams. For $\lambda \in \mathbb{Y}_{n}$ we denote by $\chi^{\lambda}$ the corresponding irreducible character (the trace of the irreducible representation of $S(n)$ indexed by $\lambda)$. Let $\operatorname{dim} \lambda=\chi^{\lambda}(e)$ be the dimension of this representation. In combinatorial terms, $\operatorname{dim} \lambda$ is the number of standard Young tableaux of shape $\lambda$. Note that for this number there exist closed expressions. Below the notation $(i, j) \in \lambda$ means that the box on the intersection of the $i$ th row and the $j$ th column belongs to $\lambda$.

Theorem. For any $n=1,2, \ldots$,

$$
\left.\chi_{z}\right|_{S(n)}=\sum_{\lambda \in \mathbb{Y}_{n}}\left(\frac{\prod_{(i, j) \in \lambda}|z+j-i|^{2}}{|z|^{2}\left(|z|^{2}+1\right) \ldots\left(|z|^{2}+n-1\right)} \frac{\operatorname{dim} \lambda}{n !}\right) \chi^{\lambda} .
$$

Note that this formula also makes sense for $z=0$.

The next claim is a direct consequence of the formula.

Proposition. The function 1 is a cyclic vector for $T_{z}$ if and only if $z \notin \mathbb{Z}$.

Thus, for nonintegral $z$, the couple $\left(T_{z}, \mathbf{1}\right)$ is a spherical representation and the character $\chi_{z}$ entirely determines $T_{z}$. 
Note that for $z=k \in \mathbb{Z}$, the cyclic span of $\mathbf{1}$ is a proper subrepresentation that "corresponds" to a particular simplex $\Omega_{p q}$ (see §3.4). Specifically,

$$
(p, q)= \begin{cases}(k, 0), & \text { if } k>0 \\ (0,|k|), & \text { if } k<0 \\ (1,1), & \text { if } k=0\end{cases}
$$

\subsection{The symmetry $z \leftrightarrow \bar{z}$}

Proposition. For any $z$, the representations $T_{z}$ and $T_{\bar{z}}$ are equivalent.

Proof. Indeed, if $z \in \mathbb{R}$ then there is nothing to prove. If $z \notin \mathbb{R}$ then $\mathbf{1}$ is cyclic, so that the claim follows from the fact that $\chi_{z}=\chi_{\bar{z}}$, which in turn is evident from Theorem of $\S 4.1$.

Note that this is by no means evident from the construction of the representations $T_{z}$.

\subsection{Disjointness}

Let $P_{z}$ be the spectral measure of the character $\chi_{z}$, see $\S 3.3$. When $z$ is integral, the measure $P_{z}$ lives on a simplex $\Omega_{p q}$, see $\S 4$.1. Now we focus on the measures $P_{z}$ with $z \notin \mathbb{Z}$.

Theorem. (i) Let $z \notin \mathbb{Z}$. Then all simplices $\Omega_{p q}$ are null sets with respect to the measure $P_{z}$.

(ii) Let $z_{1}$ and $z_{2}$ be two complex number, both nonintegral, $z_{1} \neq z_{2}$, and $z_{1} \neq \bar{z}_{2}$. Then the measures $P_{z_{1}}$ and $P_{z_{2}}$ are mutually singular.

It follows that the generalized regular representations $T_{z}$ are mutually disjoint, with the exception of the equivalence $T_{z} \sim T_{\bar{z}}$.

\subsection{A nondegeneracy property}

Proposition. All measures $P_{z}, z \in \mathbb{C}$, are supported by the subset

$$
\Omega_{0}:=\left\{(\alpha, \beta) \mid \sum \alpha_{i}+\sum \beta_{j}=1\right\} .
$$

On the contrary, the measure $P_{\infty}$ that corresponds to the biregular representation $T_{\infty}$ is the Dirac measure at the point $(\underline{0}, \underline{0})$, which is outside $\Omega_{0}$. This does not contradicts the fact that the family $\left\{T_{z}\right\}$ is a deformation of $T_{\infty}$, because $\Omega_{0}$ is dense in $\Omega$. 


\section{Determinantal point processes}

\subsection{Point configurations}

Let $\mathfrak{X}$ be a locally compact separable topological space. By a point configuration in $\mathfrak{X}$ we mean a locally finite collection $C$ of points of the space $\mathfrak{X}$. These points will also be called particles. Here "locally finite" means that the intersection of $C$ with any relatively compact subset is finite. Thus, $C$ is either finite or countably infinite. Multiple particles in $C$ are, in principle, permitted but all multiplicities must of course be finite. However, we will not really deal with configurations containing multiple particles. Let us emphasize that the particles in $C$ are unordered.

The set of all point configurations in $\mathfrak{X}$ will be denoted by $\operatorname{Conf}(\mathfrak{X})$.

\subsection{Definition of a point process}

A relatively compact Borel subset $A \subset \mathfrak{X}$ will be called a window. Given a window $A$ and $C \in \operatorname{Conf}(\mathfrak{X})$, let $\mathcal{N}_{A}(C)$ be the cardinality of the intersection $A \cap C$ (with multiplicities counted). Thus, $\mathcal{N}_{A}$ is a function on $\operatorname{Conf}(\mathfrak{X})$ taking values in $\mathbb{Z}_{+}$. We equip $\operatorname{Conf}(\mathfrak{X})$ with the Borel structure generated by the functions of the form $\mathcal{N}_{A}$.

By a measure on $\operatorname{Conf}(\mathfrak{X})$ we will mean a Borel measure with respect to this Borel structure.

By definition, a point process on $\mathfrak{X}$ is a probability measure $\mathcal{P}$ on the space $\operatorname{Conf}(\mathfrak{X})$.

In practice, point processes often arise as follows. Assume we are given a Borel space $Y$ and a map $\phi: Y \rightarrow \operatorname{Conf}(\mathfrak{X})$. The map $\phi$ must be a Borel map. i.e., for any window $A$, the superposition $\mathcal{N}_{A} \circ \phi$ must be a Borel function on $Y$. Further, assume we are given a probability Borel measure $P$ on $Y$. Then its pushforward $\mathcal{P}$ under $\phi$ is well defined and it is a point process.

Given a point process, we can speak about random point configurations $C$. Any reasonable (that is, Borel) function of $C$ becomes a random variable. For instance, $\mathcal{N}_{A}$ is a random variable for any window $A$, and we may consider the probability that $\mathcal{N}_{A}$ takes any prescribed value.

\subsection{Example: Poisson process}

Let $\rho$ be a measure on $\mathfrak{X}$. It may be infinite but must take finite values on any window. The Poisson process with density $\rho$ is characterized by the following properties:

(i) For any window $A$, the random variable $\mathcal{N}_{A}$ has the Poisson distribution with parameter $\rho(A)$, i.e.,

$$
\operatorname{Prob}\left\{\mathcal{N}_{A}=n\right\}=\frac{\rho(A)^{n}}{n !} e^{-\rho(A)}, \quad n \in \mathbb{Z}_{+} .
$$

(ii) For any pairwise disjoint windows $A_{1}, \ldots, A_{k}$, the corresponding random variables are independent. 
In particular, if $\mathfrak{X}=\mathbb{R}$ and $\rho$ is the Lebesgue measure then this is the classical Poisson process.

\subsection{Correlation measures and correlation functions}

Let $\mathcal{P}$ be a point process on $\mathfrak{X}$. One can assign to $\mathcal{P}$ a sequence $\rho_{1}, \rho_{2}, \ldots$ of measures, where, for any $n, \rho_{n}$ is a symmetric measure on the $n$-fold product $\mathfrak{X}^{n}=\mathfrak{X} \times \cdots \times \mathfrak{X}$, called the $n$-particle correlation measure. Under mild assumptions on $\mathcal{P}$ the correlation measures exist and determine $\mathcal{P}$ uniquely. They are defined as follows.

Given $n$ and a compactly supported bounded Borel function $f$ on $\mathfrak{X}^{n}$, let $\widetilde{f}$ be the function on $\operatorname{Conf}(\mathfrak{X})$ defined by

$$
\tilde{f}(C)=\sum_{i_{1}, \ldots, i_{n}} f\left(x_{i_{1}}, \ldots, x_{i_{n}}\right), \quad C=\left\{x_{1}, x_{2}, \ldots\right\} \in \operatorname{Conf}(\mathfrak{X}),
$$

summed over all $n$-tuples of pairwise distinct indices. Here we have used an enumeration of the particles in $C$ but the result does not depend on it.

Then the measure $\rho_{n}$ is characterized by the equality

$$
\int_{\mathfrak{X}^{n}} f \rho_{n}=\int_{C \in \operatorname{Conf}(\mathfrak{X})} \widetilde{f}(C) \mathcal{P}(d C),
$$

where $f$ is an arbitrary compactly supported bounded Borel function on $\mathfrak{X}^{n}$.

Examples. (i) If $\mathcal{P}$ is a Poisson process then $\rho_{n}=\rho^{\otimes n}$, where $\rho$ is the density of $\mathcal{P}$.

(ii) Assume that $\mathfrak{X}$ is discrete and $\mathcal{P}$ lives on multiplicity free configurations. Then the correlation measures say what is the probability that the random configuration contains an arbitrary given finite set of points.

Often there is a natural measure $\nu$ on $\mathfrak{X}$ (a reference measure) such that each $\rho_{n}$ has a density with respect to $\nu^{\otimes n}$. This density is called the $n$th correlation function. For instance, if $\mathfrak{X}$ is a domain of an Euclidean space and $\nu$ is the Lebesgue measure then, informally, the $n$th correlation function equals the density of the probability that the random configuration has particles in given $n$ infinitisemal regions $d x_{1}, \ldots, d x_{n}$.

\subsection{Determinantal point processes}

Let $\mathcal{P}$ be a point process on $\mathfrak{X}$. Assume that $\mathfrak{X}$ is equipped with a reference measure $\nu$ such that the correlation functions (taken with respect to $\nu$ ) exist. Let us denote these functions by $\rho_{n}\left(x_{1}, \ldots, x_{n}\right)$. The process $\mathcal{P}$ is said to be determinantal if there exists a function $K(x, y)$ on $\mathfrak{X} \times \mathfrak{X}$ such that

$$
\rho_{n}\left(x_{1}, \ldots, x_{n}\right)=\operatorname{det}\left[K\left(x_{i}, x_{j}\right)\right]_{i, j=1}^{n}, \quad n=1,2, \ldots .
$$

Then $K(x, y)$ is called a correlation kernel of $\mathcal{P}$. 
If $K(x, y)$ exists it is not unique since for any nonvanishing function $\phi(x)$ on $\mathfrak{X}$, the kernel $\phi(x) K(x, y)(\phi(y))^{-1}$ leads to the same result.

If we replace the reference measure by an equivalent one then we always can appropriately change the kernel. Specifically, if $\nu$ is multiplied by a positive function $f(x)$ then $K(x, y)$ can be replaced, say, by $K(x, y)(f(x) f(y))^{-1 / 2}$.

Examples. (i) Let $\mathfrak{X}=\mathbb{R}, \nu$ be the Lebesgue measure, and $K(x, y)=\overline{K(y, x)}$ be the kernel of an Hermitian integral operator $K$ in $L^{2}(\mathbb{R})$. Then $K(x, y)$ is a correlation kernel of a determinantal point process if and only if $0 \leq K \leq 1$ and the restriction of the kernel to any bounded interval determines a trace class operator.

(ii) The above conditions are satisfied by the sine kernel

$$
K(x, y)=\frac{\sin (\pi(x-y))}{\pi(x-y)}, \quad x, y \in \mathbb{R} .
$$

The sine kernel arises in random matrix theory. It determines a translation invariant point process on $\mathbb{R}$, which is a fundamental and probably the best known example of a determinantal point process.

\section{The point processes $\mathcal{P}_{z}$ and $\widetilde{\mathcal{P}}_{z}$. The main re- sult}

\subsection{From spectral measures to point processes}

Let $I=[-1,1] \subset \mathbb{R}$ and $I^{*}=[-1,1] \backslash\{0\}$. Let us take $I^{*}$ as the space $\mathfrak{X}$. We define an embedding $\Omega \rightarrow \operatorname{Conf}\left(I^{*}\right)$ as follows

$$
\omega=(\alpha, \beta) \mapsto C=\left\{\alpha_{i} \neq 0\right\} \cup\left\{-\beta_{j} \neq 0\right\} .
$$

That is, we remove the possible zero coordinates, change the sign of the $\beta$ coordinates, and forget the ordering. In this way we convert $\omega$ to a point configuration $C$ in the punctured segment $I^{*}$. In particular, the empty configuration $C=\varnothing$ corresponds to $\omega=(\underline{0}, \underline{0})$.

Given a probability measure $P$ on $\Omega$, its pushforward under this embedding is a probability measure $\mathcal{P}$ on $\operatorname{Conf}\left(I^{*}\right)$, i.e., a point process on the space $I^{*}$, see $\S 5.2$. Applying this procedure to the spectral measures $P_{z}(\S 4.3)$ we get point processes $\mathcal{P}_{z}$ on $I^{*}$.

\subsection{Lifting}

We aim to define a modification of the point processes $\mathcal{P}_{z}$. Fix $z \in \mathbb{C} \backslash\{0\}$ and set as usual $t=|z|^{2}$. Let $s>0$ be a random variable whose distribution has the form

$$
\frac{1}{\Gamma(t)} s^{t-1} e^{-s} d s
$$


(the gamma distribution on $\mathbb{R}_{+}$with parameter $t$.) We assume that $s$ is independent of $\mathcal{P}_{z}$. Given the random configuration $C$ of the process $\mathcal{P}_{z}$, we multiply the coordinates of all particles of $C$ by the random factor $s$. The result is a random point configuration $\widetilde{C}$ on $\mathbb{R}^{*}=\mathbb{R} \backslash\{0\}$.

We call this procedure the lifting. Under the lifting the point process $\mathcal{P}_{z}$ is transformed to a point process on $\mathbb{R}^{*}$ which we denote by $\widetilde{\mathcal{P}}_{z}$.

The lifting is in principle reversible. Indeed, due to Proposition of $\S 4.4$, we can recover $C$ from $\widetilde{C}$ by dividing all the coordinates in $\widetilde{C}$ by the sum of their absolute values.

It turns out that the lifting leads to a simplification of the initial point process.

\subsection{Transformation of the correlation functions under the lifting}

Fix the parameter $z$. Let $\rho_{n}\left(x_{1}, \ldots, x_{n}\right)$ and $\widetilde{\rho}_{n}\left(x_{1}, \ldots, x_{n}\right)$ be the correlation functions of the processes $\mathcal{P}_{z}$ and $\widetilde{\mathcal{P}}_{z}$, respectively (see $\S 5.4$ ). Here we take the Lebesgue measure as the reference measure.

The definition of the lifting implies that

$$
\tilde{\rho}_{n}\left(x_{1}, \ldots, x_{n}\right)=\int_{0}^{\infty} \frac{s^{t-1} e^{-s}}{\Gamma(t)} \rho_{n}\left(\frac{x_{1}}{s}, \ldots, \frac{x_{n}}{s}\right) \frac{d s}{s^{n}},
$$

where we agree that the function $\rho_{n}$ vanishes on $\left(\mathbb{R}^{*}\right)^{n} \backslash\left(I^{*}\right)^{n}$. Thus, the action of the lifting on the correlation functions is expressed by a ray integral transform.

This ray transform can be readily reduced to the Laplace transform. It follows that it is injective, which agrees with the fact that lifting is reversible.

\subsection{The main result}

To state the result we need some notation.

Let $W_{\kappa, \mu}(x)$ denote the Whittaker function with parameters $\kappa, \mu \in \mathbb{C}$. It is a unique solution of the differential equation

$$
W^{\prime \prime}-\left(\frac{1}{4}-\frac{\kappa}{x}+\frac{\mu^{2}-\frac{1}{4}}{x^{2}}\right) W=0
$$

with the condition $W(x) \sim x^{\kappa} e^{-\frac{x}{2}}$ as $x \rightarrow+\infty$.

This function is initially defined for real positive $x$ and then can be extended to a holomorphic function on $\mathbb{C} \backslash(-\infty, 0]$.

Next, we write $z=a+i b$ with real $a, b$ and set

$$
P_{ \pm}(x)=\frac{t^{\frac{1}{2}}}{|\Gamma(1 \pm z)|} W_{ \pm a+\frac{1}{2}, i b}(x), \quad Q_{ \pm}(x)=\frac{t^{\frac{3}{2}} x^{-\frac{1}{2}}}{|\Gamma(1 \pm z)|} W_{ \pm a-\frac{1}{2}, i b}(x) .
$$


Main Theorem. For any $z \in \mathbb{C} \backslash\{0\}$, the point process $\widetilde{\mathcal{P}}_{z}$ is a determinantal process whose correlation kernel can be written as

$$
K(x, y)= \begin{cases}\frac{P_{+}(x) Q_{+}(y)-Q_{+}(x) P_{+}(y)}{x-y}, & x>0, y>0 \\ \frac{P_{+}(x) P_{-}(-y)+Q_{+}(x) Q_{-}(-y)}{x-y}, & x>0, y<0 \\ \frac{P_{+}(x) P_{+}(y)+Q_{-}(-x) Q_{+}(y)}{x-y}, & x<0, y>0 \\ -\frac{P_{-}(-x) Q_{-}(-y)-Q_{-}(-x) P_{-}(-y)}{x-y}, & x<0, y<0\end{cases}
$$

where $x, y \in \mathbb{R}^{*}$ and the indeterminacy arising for $x=y$ is resolved via the L'Hospital rule.

We call the kernel $K(x, y)$ the Whittaker kernel.

Note that $K(x, y)$ is real valued. It is not symmetric but satisfies the symmetry property

$$
K(x, y)=\operatorname{sgn}(x) \operatorname{sgn}(y) K(y, x),
$$

where $\operatorname{sgn}(x)$ equals \pm 1 according to the sign of $x$. This property can be called $J$-symmetry, it means that the kernel is symmetric with respect to an indefinite inner product.

\subsection{The $L$-operator}

Split the Hilbert space $L^{2}\left(\mathbb{R}^{*}\right)$ into the direct sum $L^{2}\left(\mathbb{R}_{+}\right) \oplus L^{2}\left(\mathbb{R}_{-}\right)$, where all $L^{2}$ spaces are taken with respect to the Lebesgue measure. According to this splitting we will write operators in $L^{2}\left(\mathbb{R}^{*}\right)$ in block form, as $2 \times 2$ operator matrices.

Let

$$
L=\left[\begin{array}{cc}
0 & A \\
-A^{t} & 0
\end{array}\right]
$$

where $A$ is the integral operator with the kernel

$$
A(x, y)=\left|\frac{\sin (\pi z)}{\pi}\right|^{2} \cdot \frac{\left(\frac{x}{|y|}\right)^{\operatorname{Re} z} e^{-\frac{x-y}{2}}}{x-y}, \quad x>0, \quad y<0 .
$$

By $A^{t}$ we denote the conjugate operator $L^{2}\left(\mathbb{R}_{+}\right) \rightarrow L^{2}\left(\mathbb{R}_{-}\right)$.

Theorem. Assume that $-\frac{1}{2}<\Re z<\frac{1}{2}, z \neq 0$. Then $A$ is a bounded operator $L^{2}\left(\mathbb{R}_{-}\right) \rightarrow L^{2}\left(\mathbb{R}_{+}\right)$and the correlation kernel $K(x, y)$ is the kernel of the operator $L(1+L)^{-1}$.

Note that, in contrast to $K$, the kernel of $L$ does not involve special functions. 


\subsection{An application}

Fix $z \in \mathbb{C} \backslash \mathbb{Z}$ and consider the probability space $\left(\Omega, P_{z}\right)$. For any $k=1,2, \ldots$ the coordinates $\alpha_{k}$ and $\beta_{k}$ are functions in $\omega \in \Omega$, hence we may view them as random variables. The next result provides an information about the rate of their decay as $i, j \rightarrow \infty$.

Theorem. With probability 1, there exist limits

$$
\lim _{k \rightarrow \infty}\left(\alpha_{k}\right)^{\frac{1}{k}}=\lim _{k \rightarrow \infty}\left(\beta_{k}\right)^{\frac{1}{k}}=q(z) \in(0,1),
$$

where

$$
q(z)=\exp \left(\pi \frac{\operatorname{ctg} \pi z-\operatorname{ctg} \pi \bar{z}}{z-\bar{z}}\right)=\exp \left(-\sum_{n \in \mathbb{Z}} \frac{1}{|z-n|^{2}}\right)
$$

\section{Scheme of the proof of the Main Theorem}

\subsection{The $\mathrm{z}-$-measures}

Recall that by $\mathbb{Y}_{n}$ we denote the finite set of Young diagrams with $n$ boxes. Set

$$
P_{z}^{(n)}(\lambda)=\frac{\prod_{(i, j) \in \lambda}|z+j-i|^{2}}{|z|^{2}\left(|z|^{2}+1\right) \ldots\left(|z|^{2}+n-1\right)} \frac{(\operatorname{dim} \lambda)^{2}}{n !}, \quad \lambda \in \mathbb{Y}_{n} .
$$

Comparing this with the expression of $\left.\chi_{z}\right|_{S(n)}(\S 4.1)$ we see that the quantities $P_{z}^{(n)}(\lambda)$ are the coefficients in the expansion of $\chi_{z}$ in the normalized irreducible characters $\chi^{\lambda} / \operatorname{dim} \lambda$. It follows that

$$
\sum_{\lambda \in \mathbb{Y}_{n}} P_{z}^{(n)}(\lambda)=1
$$

Thus, for any fixed $n=1,2, \ldots$, the quantities $P_{z}^{(n)}(\lambda)$ determine a probability measure on $\mathbb{Y}_{n}$. We will denote it by $P_{z}^{(n)}$ and call it the $z$-measure on $Y_{n}$.

\subsection{Frobenius coordinates and the embedding $\mathbb{Y}_{n} \hookrightarrow \Omega$}

Given $\lambda \in \mathbb{Y}_{n}$, let $\lambda^{\prime}$ be the transposed diagram and $d$ be the number of diagonal boxes in $\lambda$. We define the modified Frobenius coordinates of $\lambda$ as

$$
a_{i}=\lambda_{i}-i+\frac{1}{2}, \quad b_{i}=\lambda_{i}^{\prime}-i+\frac{1}{2}, \quad i=1, \ldots, d .
$$

Note that

$$
a_{1}>\cdots>a_{d}>0, \quad b_{1}>\cdots>b_{d}>0, \quad \sum_{i=1}^{d}\left(a_{i}+b_{i}\right)=n .
$$


For any $n=1,2, \ldots$ we embed $\mathbb{Y}_{n}$ into $\Omega$ by making use of the map

$$
\begin{gathered}
\lambda \mapsto \omega_{\lambda}=(\alpha, \beta), \\
\alpha=\left(\frac{a_{1}}{n}, \ldots, \frac{a_{d}}{n}, 0,0, \ldots\right), \quad \beta=\left(\frac{b_{1}}{n}, \ldots, \frac{b_{d}}{n}, 0,0, \ldots\right) .
\end{gathered}
$$

As $n \rightarrow \infty$, the points $\omega_{\lambda}$ coming from the diagrams $\lambda \in \mathbb{Y}_{n}$ fill out the space $\Omega$ more and more densely. Thus, for large $n$, the image of $\mathbb{Y}_{n}$ in $\Omega$ can be viewed as a discrete approximation of $\Omega$.

\subsection{Approximation of $P_{z}$ by $\mathbf{z}$-measures}

Let $\underline{P}_{z}^{(n)}$ be the pushforward of the measure $P_{z}^{(n)}$ under the embedding $\mathbb{Y}_{n} \hookrightarrow \Omega$. This is a probability measure on $\Omega$.

Approximation Theorem. As $n \rightarrow \infty$, the measures $\underline{P}_{z}^{(n)}$ weakly converge to the measure $P_{z}$.

This fact is the starting point for explicit computations related to the measures $P_{z}$.

\subsection{The mixed $\mathrm{z}-$-measures}

Let $\mathbb{Y}=\mathbb{Y}_{0} \cup \mathbb{Y}_{1} \cup \mathbb{Y}_{2} \cup \ldots$ be the set of all Young diagrams. We agree that $\mathbb{Y}_{0}$ consists of a single element - the empty diagram $\varnothing$. Fix $z \in \mathbb{C} \backslash\{0\}$ and $\xi \in(0,1)$. We define a measure $\widetilde{P}_{z, \xi}$ on $\mathbb{Y}$ as follows:

$$
\widetilde{P}_{z, \xi}(\lambda)=P_{z}^{(n)}(\lambda) \cdot(1-\xi)^{|z|^{2}} \frac{|z|^{2}\left(|z|^{2}+1\right) \ldots\left(|z|^{2}+n-1\right)}{n !} \xi^{n}, \quad \lambda \in \mathbb{Y},
$$

where $n$ is the number of boxes in $\lambda$ and $P_{z}^{(0)}(\varnothing):=1$.

In other words, $\widetilde{P}_{z, \xi}$ is obtained by mixing together all the $\mathrm{z}-$ measures $P_{z}^{(0)}, P_{z}^{(1)}, \ldots$, where the weight of the $n$th component is equal to

$$
\pi_{t, \xi}(n)=(1-\xi)^{t} \frac{t(t+1) \ldots(t+n-1)}{n !} \xi^{n}, \quad t=|z|^{2} .
$$

Note that

$$
\sum_{n=0}^{\infty} \pi_{t, \xi}(n)=1
$$

It follows that $\widetilde{P}_{z, \xi}$ is a probability measure. Let us call it the mixed $z$-measure.

Note that, as $z \rightarrow 0$, the measure $\widetilde{P}_{z, \xi}$ tends to the Dirac mass at $\{\varnothing\}$ for any fixed $\xi$. 


\subsection{The lattice process $\widetilde{\mathcal{P}}_{z, \xi}$}

Set

$$
\mathbb{Z}^{\prime}=\mathbb{Z}+\frac{1}{2}=\left\{\ldots,-\frac{3}{2},-\frac{1}{2}, \frac{1}{2}, \frac{3}{2}, \ldots\right\} .
$$

Using the notation of $\S 7.2$ we assign to an arbitrary Young diagram a point configuration $C \in \operatorname{Conf}\left(\mathbb{Z}^{\prime}\right)$, as follows

$$
\lambda \mapsto C=\left\{-b_{1}, \ldots,-b_{d}, a_{d}, \ldots, a_{1}\right\} .
$$

The correspondence $\lambda \mapsto C$ defines an embedding $\mathbb{Y} \hookrightarrow \operatorname{Conf}\left(\mathbb{Z}^{\prime}\right)$. Take the pushforward of the measure $P_{z, \xi}$ under this embedding. It is a probability measure on $\operatorname{Conf}\left(\mathbb{Z}^{\prime}\right)$, hence a point process on $\mathbb{Z}^{\prime}$. Let us denote it by $\widetilde{\mathcal{P}}_{z, \xi}$.

Theorem. The process $\widetilde{\mathcal{P}}_{z, \xi}$ on the lattice $\mathbb{Z}^{\prime}$ is determinantal. Its correlation kernel can be explicitly computed: it has the form quite similar to that of the kernel $K(x, y)$ from $\S 6.4$, where the corresponding functions $P_{ \pm}$and $Q_{ \pm}$are now expressed through the Gauss hypergeometric function.

\subsection{Idea of proof of the Main Theorem}

Given $\xi \in(0,1)$, we embed the lattice $\mathbb{Z}^{\prime}$ into $\mathbb{R}^{*}$ as follows

$$
\mathbb{Z}^{\prime} \ni x \mapsto(1-\xi) x \in \mathbb{R}^{*} .
$$

Let $\widetilde{\mathcal{P}}_{z, \xi}$ be the pushforward of $\widetilde{\mathcal{P}}_{z, \xi}$ under this embedding. We can view $\widetilde{\mathcal{P}}_{z, \xi}$ as a point process on $\mathbb{R}^{*}$.

Remark that the probability distribution $\pi_{t, \xi}$ on $\mathbb{Z}_{+}$introduced in $\S 7.4$ approximates in an appropriate scaling limit as $\xi \nearrow 1$ the gamma distribution on $\mathbb{R}^{*}$ with parameter $t$. Specifically, the scaling has the form $n \mapsto(1-\xi) n$. Recall that we have used the gamma distribution in the definition of the lifting, see $\S 6.2$.

Combining this fact with the Approximation Theorem of $\S 7.3$ we conclude that the process $\widetilde{\mathcal{P}}_{z, \xi}$ must converge to the process $\widetilde{\mathcal{P}}_{z}$ as $\xi \nearrow 1$ in a certain sense. More precisely, we prove that the correlation measures of the former process converge to the respective correlation measures of the latter process.

On the other hand, we can explicitly compute the scaled limit of the lattice correlation measures using the explicit expression of the lattice kernel from $\S 7.5$. It turns out that then the Gauss hypergeometric function degenerates to the Whittaker function and we get the formulas of $\S 6.4$.

\section{Notes and references}

\subsection{Section 1}

The main reference to this section is the paper Kerov-Olshanski-Vershik [41]. 
§1.1. Peter-Weyl's theorem is included in many textbooks on representation theory. See, e.g., Naimark [55], §32.

$\S 1.2$. From the purely algebraic point of view, there is no single infinite analog of the permutation groups $S(n)$ but a number of different versions. The group $S(\infty)=\lim S(n)$ formed by finite permutations of the set $\{1,2, \ldots\}$ and the group of all permutations of this set may be viewed as the minimal and the maximal versions. There is also a huge family of intermediate groups. The choice of an appropriate version may vary depending on the applications we have in mind. Certain topological groups connected with $S(\infty)$ are discussed in Olshanski 61, Okounkov [56, [57].

§1.3. The result of the Proposition is closely related to von Neumann's classical construction of $\mathrm{II}_{1}$ factors. See Murray-von Neumann [51, ch. 5, and Naimark [55], ch. VII, §38.5.

$\S 1.4$. The $G$-space $\mathfrak{S}$ of virtual permutations was introduced in KerovOlshanski-Vershik 41. Notice that the canonical projection $p_{n}$ emerged earlier, see Aldous [1, p. 92. A closely related construction, which also appeared earlier, is the so-called Chinese restaurant process, see, e.g., Arratia-Barbour-Tavaré [2], $\S 2$ and references therein. Projective limit constructions for classical groups and symmetric spaces are considered in Pickrell [71, Neretin [53, Olshanski 64]. Earlier papers: Hida-Nomoto [29, Yamasaki [90, 91], Shimomura 73].

$\S 1.5$. The definition of the Ewens measures $\mu_{t}$ on the space $\mathfrak{S}$ was proposed in [41, see also Kerov-Tsilevich 42], Kerov [37] (the latter paper deals with a generalization of these measures). The definition of 41] was inspired by the fundamental concept of the Ewens sampling formula, which was derived in 1972 by Ewens [26] in the context of population genetics. There is a large literature concerning Ewens' sampling formula (or Ewens' partition structure). See, e.g., the papers Watterson [89, Kingman [44, 45], 47], Arratia-Barbour-Tavaré [2], 3], Ewens [27, which contain many other references.

\$1.6. The results were established in [41. For projective limits of classical groups and symmetric spaces, there also exist distinguished families of measures with good transformation properties, see Pickrell [71], Neretin [53], Olshanski 64.

\$1.7. The representations $T_{z}$ were introduced in [41]. A parallel construction exists for infinite-dimensional classical groups and symmetric spaces, see the pioneer paper Pickrell [71] and also Neretin [53, Olshanski [64].

\subsection{Section 2}

$\S 2.1$. The concept of spherical representations is usually employed for Gelfand pairs $(\mathcal{G}, \mathcal{K})$. According to the conventional definition, $(\mathcal{G}, \mathcal{K})$ is said to be a Gelfand pair if the subalgebra of $\mathcal{K}$-biinvariant functions in the group algebra $L^{1}(\mathcal{G})$ is commutative. This works for locally compact $\mathcal{G}$ and compact $\mathcal{K}$. There

exists, however, a reformulation which makes sense for arbitrary groups, see Olshanski 62. Our pair $(G, K)$ is a Gelfand pair, see Olshanski 63.

$\S 2.2$. For general facts concerning positive definite functions on groups, see, e.g., Naimark [55]. 
§2.3. There exist at least two different ways to define characters for infinitedimensional representations. The most known recipe (Gelfand, Harish-Chandra) is to view characters not as ordinary functions but as distributions on the group. This idea works perfectly for a large class of Lie groups and $p$-adic groups but not for groups like $S(\infty)$. The definition employed here follows another approach, which goes back to von Neumann. Extreme characters of a group $\mathcal{K}$ are related to finite factor representations of $\mathcal{K}$ in the sense of von Neumann. See Thoma [76], 77], Stratila-Voiculescu [75], Voiculescu [87.

§2.4. The correspondence between extreme characters and irreducible spherical representations was pointed out in Olshanski [61, 62. The Proposition follows from the fact that our pair $(G, K)$ is a Gelfand pair, see Olshanski 63 .

The irreducible spherical representations of $(G, K)$ form a subfamily of a larger family of representations called admissible representations, see Olshanski 61, 62, 63. On the other hand, aside from finite factor representations of $S(\infty)$ that correspond to extreme characters, there exist interesting examples of factor representations of quite different nature, see Stratila-Voiculescu [75].

Explicit realizations of finite factor representations of $S(\infty)$ and irreducible spherical representations of $(G, K)$ are given in Vershik-Kerov [82, Wassermann [88, Olshanski 63].

$\S 2.5$. There are various methods to establish the existence and uniqueness of the spectral decomposition. See, e.g., Diaconis-Freedman 23, Voiculescu [87, Olshanski 64]. One more approach, which is specially adapted to the group $S(\infty)$ and provides an explicit description of $\operatorname{Ex}(\mathcal{X}(S(\infty)))$, is proposed in Kerov-Okounkov-Olshanski [39].

\subsection{Section 3}

$\S 3.1$. The expressions $p_{k}(\alpha, \beta)$ are supersymmetric analogs of power sums. About the role of supersymmetric functions in the theory of characters of $S(\infty)$ see Vershik-Kerov [83], Olshanski-Regev-Vershik 65].

$\S 3.2$. The Thoma set $\Omega$ can be viewed as an infinite-dimensional simplex. The subsets $\Omega_{p q}$ are exactly its finite-dimensional faces.

§3.3. Thoma's paper [76] was the first work about characters of $S(\infty)$. It contains the classification of extreme characters (Thoma's theorem), which was obtained using complex-analytic tools. Thoma's theorem is equivalent to another classification problem - that of one-sided totally positive sequences. Much earlier, that problem was raised by Schoenberg and solved by Edrei [25]. The equivalence of both problems was implicit in Thoma's paper [76] but Thoma apparently was not aware of the works on total positivity.

The next step was made by Vershik and Kerov [83. Following a general principle earlier suggested in Vershik [79], Vershik and Kerov found a new proof of Thoma's theorem. Their approach is based on studying the limit transition from characters of $S(n)$ to characters of $S(\infty)$. This provides a very natural interpretation of Thoma's parameters $\alpha_{i}, \beta_{j}$.

Developing further the asymptotic approach of [83, Kerov-Okounkov-Olshanski [39] obtained a generalization of Thoma's theorem. An even more general claim 
was conjectured by Kerov in 35.

One of the fruitful ideas contained in Vershik-Kerov's paper 83 concerns the combinatorics of irreducible characters $\chi^{\lambda}$ of the finite symmetric groups. Assume that $\lambda \in \mathbb{Y}_{n}$ and $\rho$ is a partition of $m$, where $m \leq n$. Let $\chi_{\rho}^{\lambda}$ denote the value of $\chi^{\lambda}$ at the conjugacy class in $S(n)$ indexed by the partition $\rho \cup 1^{n-m}$ of $n$. The idea was to consider $\chi_{\rho}^{\lambda}$ as a function in $\lambda$ with $\rho$ viewed as a parameter. Vershik and Kerov discovered that the function $\lambda \mapsto \chi_{\rho}^{\lambda}$, after a simple normalization, becomes a supersymmetric function in the modified Frobenius coordinates of $\lambda$. This function is inhomogeneous and its top degree homogeneous term is the supersymmetric (product) power sum indexed by $\rho$. Further results in this directions: Kerov-Olshanski [40, Okounkov-Olshanski 60], Olshanski-Regev-Vershik [65]. Even in the simplest case when $\rho$ consists of a single part $(\rho=(m))$ the function $\lambda \mapsto \chi_{\rho}^{\lambda}=\chi_{(m)}^{\lambda}$ is rather nontrivial. See Wassermann [88, Kerov [36], Biane [5], Ivanov-Olshanski [32].

§3.4. The spectral decomposition of $T_{z}$ 's for integral values of $z$ was obtained in Kerov-Olshanski-Vershik 41.

\subsection{Section 4}

$\S 4.1$. The results were obtained in Kerov-Olshanski-Vershik 41]. Similar results for other groups: Pickrell [71, Olshanski 64.

$\S 4.2$. One can define intertwining operators for the representations $T_{z}$ and $T_{\bar{z}}$. These operators have interesting properties. See Kerov-Olshanski-Vershik [11.

§4.3. The result was obtained in Kerov-Olshanski-Vershik [41]. Note that the Theorem of $\S 6.6$ implies a weaker result: the spectral measures $P_{z_{1}}$ and $P_{z_{2}}$ are mutually singular for any $z_{1}, z_{2} \in \mathbb{C} \backslash \mathbb{Z}$ such that $q\left(z_{1}\right) \neq q\left(z_{2}\right)$.

$\S 4.4$. The result was announced in Kerov-Olshanski-Vershik [1]. It can be proved in different ways, see Olshanski [6], Borodin 67].

\subsection{Section 5}

$\S \S 5.1-5.4$. The material is standard. See Daley and Vere-Jones [21], Lenard 48, Kingman 47. Point processes are also called random point fields.

§5.5. The class of determinantal point process was first singled out by Macchi [49, [50] under the name of fermion processes. The motivation comes from a connection with the fermionic Fock space. The term "determinantal" was suggested in Borodin-Olshanski [15. We found it more appropriate, because in our concrete situation, point configurations may be viewed as consisting of particles of two opposite charges. A number of important examples of determinantal point processes emerged in random matrix theory, see, e.g., Dyson [24], Mehta [52, Nagao-Wadati [54], Tracy-Widom 78], and the references therein. However, to our knowledge, up to the recent survey paper by Soshnikov [74], the experts in this field did not pay attention to general properties of determinantal processes and did not introduce any general name for them.

The result stated in Example (i) is due to Soshnikov [74]. 


\subsection{Section 6}

$\S 6.1$. The spectral measures $P_{z}$ with nonintegral parameter $z$ originally looked mysterious: it was unclear how to handle them.

The idea of converting the measures $P_{z}$ into point processes $\mathcal{P}_{z}$ and computing the correlation functions was motivated by the following observation. It turns out that the coefficients of the expansion of $\S 4.1$ can be interpreted as moments of certain auxiliary measures (we called them the controlling measures). The controlling measures are determined by these moments uniquely. On the other hand, the correlation functions can be expressed through the controlling measures. It follows that evaluating the correlation functions can be reduced to solving certain (rather complicated) multidimensional moment problems.

We followed first this way (see the preprints [66]- 6 ;0; part of results was published in Borodin [7, 8]; a summary is given in Borodin-Olshanski [14]). A general description of the method and the evaluation of the first correlation function are given in Olshanski [66. In Borodin [7] the moment problem in question is studied in detail. This leads (Borodin [67]) to some formulas for the higher correlation functions: a multidimensional integral representation and an explicit expression through a multivariate Laurichella hypergeometric series of type B. Both are rather involved.

$\S \S 6.2-6.3$. The idea of lifting (Borodin [69]) turned out to be extremely successful, because it leads to a drastic simplification of the correlation functions. What is even more important is that due to this procedure we finally hit a nice class of point processes, the determinantal ones.

§6.4. The derivation of the Whittaker kernel by the first method is given in Borodin 69, 88. It should be noted that the Whittaker kernel belongs to the class of integrable kernels. This class was singled out by Its-Izergin-KorepinSlavnov [31, see also Deift [22, Borodin [11].

§6.5. The claim concerning the L-operator and some related facts are contained in Olshanski [70]. A conclusion is that (at least when $|\Re z|<1 / 2$ ) the whole information about the spectral measure $P_{z}$ is encoded in a very simple kernel $L(x, y)$.

§6.6. The result is obtained in Borodin-Olshanski 68. It can be viewed as a strong law of large numbers. Roughly speaking, the coordinates $\alpha_{k}, \beta_{k}$ decay like the terms of the geometric progression $\left\{q(z)^{k}\right\}$. A similar result holds for point processes of quite different type (Poisson-Dirichlet distributions), see Vershik-Shmidt 86.

Notice that the preprints [66]-70] contain a number of other results, some of them remain still unpublished.

\section{7 $\quad$ Section 7}

The main reference for this section is the paper Borodin-Olshanski [15, which gives an alternate way of proving the Main Theorem. The method of [15] is simpler than the previous approach based on a moment problem. Furthermore, our second approach explains the origin of the lifting. However, the correlation 
functions for the initial process $\mathcal{P}_{z}$ are not directly obtained in this way.

§7.1. The z-measures $P_{z}^{(n)}$ with fixed parameter $z$ and varying index $n$ satisfy the coherency relation

$$
P_{z}^{(n)}(\mu)=\sum_{\lambda \in \mathbb{Y}_{n+1}: \lambda \supset \mu} \frac{\operatorname{dim} \mu}{\operatorname{dim} \lambda} P_{z}^{(n+1)}(\lambda), \quad n=1,2, \ldots, \quad \mu \in \mathbb{Y}_{n} .
$$

It expresses the fact that the function $\left.\chi_{z}\right|_{S(n+1)}$ is an extension of the function $\left.\chi_{z}\right|_{S(n)}$. The coherency relation is not evident from the explicit expression for the $\mathrm{z}$-measures.

As $|z| \rightarrow \infty$, the measures $P_{z}^{(n)}$ converge to the Plancherel measure on $\mathbb{Y}_{n}$,

$$
P_{\infty}^{(n)}(\lambda)=\frac{(\operatorname{dim} \lambda)^{2}}{n !} .
$$

Note that the expression for $P_{z}^{(n)}(\lambda)$ looks as a product over the boxes of $\lambda$ times $P_{\infty}^{(n)}(\lambda)$. This property together with the coherency relation can be used for a combinatorial characterization of the $z$-measures, see Rozhkovskaya [72].

Actually, the term " $\mathrm{z}-$ measures" has a somewhat wider meaning: the family $\left\{P_{z}^{(n)}\right\}$ forms the "principal series" while the whole family of the $\mathrm{z}$-measures also includes a "complementary series" and a "degenerate series" of measures which are given by similar expressions.

A much larger family of Schur measures was introduced by Okounkov [59]. In general, the Schur measures do not obey the coherency relation and hence do not correspond to characters of $S(\infty)$. However, they also give rise to determinantal point processes. It would be interesting to know whether the z-measures exhaust all Schur measures satisfying the coherency relation.

Kerov [38] introduced analogs of $\mathrm{z}$-measures satisfying a certain one-parameter deformation of the coherency relation (the coherency relation written above is closely related to the Schur functions, while Kerov's more general form of the coherency relation is related to the Jack symmetric functions, see also KerovOkounkov-Olshanski [39]). For another approach, see Borodin-Olshanski [16]. Study of the point processes corresponding to these more general $\mathrm{z}$-measures was started in Borodin-Olshanski [20].

An analog of $\mathrm{z}$-measures corresponding to projective characters of $S(\infty)$ was found in Borodin 6]. See also Borodin-Olshanski [16].

The paper Borodin-Olshanski [17] presents a survey of connections between $\mathrm{z}$-measures and a number of models arising in combinatorics, tiling, directed percolation and random matrix theory.

\$7.2. The idea of embedding $\mathbb{Y}$ into $\Omega$ is due to Vershik and Kerov [83. In a more general context it is used in Kerov-Okounkov-Olshanski [39].

§7.3. The Approximation Theorem actually holds for spectral measures corresponding to arbitrary characters of $S(\infty)$. See Kerov-Okounkov-Olshanski [39].

\$7.4. What we called "mixing" is a well-known trick. Under different names it is used in various asymptotic problems of combinatorics and statistical physics. See, e.g., Vershik [1]. The general idea is to replace a large $n$ 
limit, where the index $n$ enumerates different probabilistic ensembles, by a limit transition of another kind (we are dealing with a unifying ensemble depending on a parameter and let the parameter tend to a limit). In many situations the two limit transitions lead to the same result. For instance, this usually happens for the poissonization procedure, when the mixing distribution on $\mathbb{Z}_{+}$is a Poisson distribution. (About the poissonized Plancherel measure, see BaikDeift-Johansson 4, Borodin-Okounkov-Olshanski [13, Johansson [33.) A key property of the Poisson distribution is that as its parameter goes to infinity, the standard deviation grows more slowly than the mean. In our situation, instead of Poisson we have to deal with the distribution $\pi_{t, \xi}$, a particular case of the negative binomial distribution. As $\xi \nearrow 1$, the standard deviation and the mean of $\pi_{t, \xi}$ have the same order of growth, which results in a nontrivial transformation of the large $n$ limit (the lifting).

§7.5. The fact that the lattice process $\widetilde{\mathcal{P}}_{z}$ is determinantal is checked rather easily. The difficult part of the Theorem is the calculation of the correlation kernel. This can be done in different ways, see Borodin-Olshanski [15, Okounkov [58, [59. Borodin [9], 11] describes a rather general procedure of computing correlation kernels via a Riemann-Hilbert problem.

$\S 7.6$. For more details see Borodin-Olshanski [15].

\subsection{Other problems of harmonic analysis leading to point processes}

A parallel but more complicated theory holds for the infinite-dimensional unitary group $U(\infty)=\lim U(N)$. For this group, there exists a completion $\mathfrak{U}$ of the group space $U(\infty)$, which plays the role of the space $\mathfrak{S}$ of virtual permutations. On $\mathfrak{U}$, there exists a family of measures with good transformation properties which give rise to certain unitary representations of $U(\infty) \times U(\infty)$ - analogs of the representations $T_{z}$. See Neretin [53], Olshanski [64. The problem of harmonic analysis for these representations is studied in Borodin-Olshanski [19]. It leads to determinantal point processes on the space $\mathbb{R} \backslash\left\{ \pm \frac{1}{2}\right\}$. Their correlation kernels were found in [19]: these are integrable kernels expressed through the Gauss hypergeometric function.

There exists a similarity between decomposition of unitary representations into irreducible ones and decomposition of invariant measures on ergodic components. Both problems often can be interpreted in terms of barycentric decomposition on extreme points in a convex set. Below we briefly discuss two problems of "harmonic analysis for invariant measures" that lead to point processes.

The first problem concerns invariant probability measures for the action of the diagonal group $K \subset G$ on the space $\mathfrak{S}$. Recall that $K$ is isomorphic to $S(\infty)$. Such measures are in 1-1 correspondence with partition structures in the sense of Kingman [43]. The set of all $K$-invariant probability measures on $\mathfrak{S}$ (or partition structures) is a convex set. Its extreme points correspond to ergodic invariant measures whose complete classification is due to Kingman [4], [46], see also Kerov [34. Kingman's result is similar to Thoma's theorem. The de- 
composition of Ewens' measures $\mu_{t}$ on ergodic components leads to a remarkable one-parameter family of point processes on $(0,1]$ known as Poisson-Dirichlet distributions. There is a large literature on Poisson-Dirichlet distributions, we cite only a few works: Watterson [89, Griffiths [28], Vershik-Shmidt [86], Ignatov [30, Kingman [43, 44, 47], Vershik [80, Arratia-Barbour-Tavaré 3]. One can show that the lifting of the Poisson-Dirichlet distribution with parameter $t>0$ is the Poisson process on $(0,+\infty)$ with density $\frac{t}{x} e^{-x} d x$.

In the second problem, one deals with $(U(\infty), \mathfrak{U})$ instead of $(S(\infty), \mathfrak{S})$. Here we again have a distinguished family of invariant measures, see BorodinOlshanski [18, Olshanski 64]. Their decomposition on ergodic components is described in terms of certain determinantal point processes on $\mathbb{R}^{*}$. The corresponding correlation kernels are integrable and are expressed through another solution of Whittaker's differential equation $(\$ 6.4)$, see [18]. This subject is closely connected with Dyson's unitary circular ensemble, see [18], 64].

For the point processes mentioned above, a very interesting quantity is the position of the rightmost particle in the random point configuration. In the Poisson-Dirichlet case, the distribution of this random variable is given by a curious piece-wise analytic function satisfying a linear difference-differential equation: see Vershik-Shmidt 86], Watterson [89. For the (discrete and continuous) determinantal point processes arising in harmonic analysis, the distribution of the rightmost particle can be expressed through solutions of certain nonlinear (difference or differential) Painlevé equations: see Borodin 10, Borodin-Deift 12 .

\section{References}

[1] D. J. Aldous, Exchangeability and related topics, in: Springer Lecture Notes in Math. 1117 (1985), pp. 2-199.

[2] R. Arratia, A. D. Barbour, and S. Tavaré, Poisson processes approximations for the Ewens sampling formula, Ann. Appl. Probab., 2 (1992), 519-535.

[3] R. Arratia, A. D. Barbour, and S. Tavaré, Random combinatorial structures and prime factorizations, Notices Amer. Math. Soc., 44 (1997), no. 8, 903910 .

[4] J. Baik, P. Deift and K. Johansson, On the distribution of the length of the longest increasing subsequence of random permutations, J. Amer. Math. Soc., 12 (1999), no. 4, 1119-1178.

[5] Ph. Biane, Representations of symmetric groups and free probability, Advances in Math., 138 (1998), 126-181.

[6] A. Borodin, Multiplicative central measures on the Schur graph, in: Representation theory, dynamical systems, combinatorial and algorithmic methods II (A. M. Vershik, ed.), Zapiski Nauchnykh Seminarov POMI 240, 
Nauka, St. Petersburg, 1997, 44-52 (Russian); English translation: J. Math. Sci. (New York), 96 (1999), no. 5, 3472-3477.

[7] A. Borodin, Characters of symmetric groups and correlation functions of point processes, Funktsional. Anal. Prilozhen., 34 (2000), no. 1, 12-28 (Russian); English translation: Funct. Anal. Appl. 34 (2000), no. 1, 10-23.

[8] A. Borodin, Harmonic analysis on the infinite symmetric group and the Whittaker kernel, Algebra Anal. 12 (2001), no. 5, 28-63 (Russian); English translation: St. Petersburg Math. J., 12 (2001), no. 5, 733-759.

[9] A. Borodin, Riemann-Hilbert problem and the discrete Bessel kernel, Intern. Math. Research Notices, 2000:9 (2000), 467-494; math/9912093.

[10] A. Borodin, Discrete gap probabilities and discrete Painlevé equations, Duke Math. J, to appear; math-ph/0111008

[11] A. Borodin, Asymptotic representation theory and Riemann-Hilbert problem, in this volume; math/0110318

[12] A. Borodin and P. Deift, Fredholm determinants, Jimbo-Miwa-Ueno taufunctions, and representation theory, Comm. Pure Appl. Math. 55 (2002), no. 9, 1160-1230; math-ph/0111007

[13] A. Borodin, A. Okounkov and G. Olshanski, Asymptotics of Plancherel measures for symmetric groups, J. Amer. Math. Soc., 13 (2000), no. 3, 481-515; math/9905032

[14] A. Borodin and G. Olshanski, Point processes and the infinite symmetric group, Math. Research Lett., 5 (1998), 799-816; math/9810015

[15] A. Borodin and G. Olshanski, Distributions on partitions, point processes, and the hypergeometric kernel, Commun. Math. Phys., 211 (2000), 335358 ; math/9904010

[16] A. Borodin and G. Olshanski, Harmonic functions on multiplicative graphs and interpolation polynomials, Electronic J. Comb., 7 (2000), paper \#R28; math/9912124

[17] A. Borodin and G. Olshanski, Z-Measures on partitions, RobinsonSchensted-Knuth correspondence, and $\beta=2$ random matrix ensembles, in: Random matrix models and their applications (P. M. Bleher and A. R. Its, eds). Mathematical Sciences Research Institute Publications 40, Cambridge Univ. Press, 2001, 71-94; math/9905189.

[18] A. Borodin and G. Olshanski, Infinite random matrices and ergodic measures, Comm. Math. Phys., 223 (2001), 87-123; math-ph/0010015.

[19] A. Borodin and G. Olshanski, Harmonic analysis on the infinitedimensional unitary group and determinantal point processes, Ann. Math, to appear; math/0109194 
[20] A. Borodin and G. Olshanski, Z-measures on partitions and their scaling limits, 2002, math-ph/0210148

[21] D. J. Daley and D. Vere-Jones, An introduction to the theory of point processes, Springer Series in Statistics, Springer, 1988.

[22] P. Deift, Integrable operators, in: Differential operators and spectral theory: M. Sh. Birman's 70th anniversary collection (V. Buslaev, M. Solomyak, D. Yafaev, eds.), American Mathematical Society Translations, ser. 2, vol. 189, Providence, R.I., Amer. Math. Soc., 1999.

[23] P. Diaconis and D. Freedman, Partial exchangeability and sufficiency, in: Statistics: Applications and New Directions (Calcutta, 1981), Indian Statist. Inst., Calcutta, 1984, 205-236.

[24] F. J. Dyson, Statistical theory of the energy levels of complex systems I, II, III, J. Math. Phys., 3 (1962), 140-156, 157-165, 166-175.

[25] A. Edrei, On the generating functions of totally positive sequences II, J. Analyse Math., 2 (1952), 104-109.

[26] W. J. Ewens, The sampling theory of selectively neutral alleles, Theoret. Population Biology, 3 (1972), 87-112.

[27] W. J. Ewens, Population Genetics Theory - the Past and the Future, in: Mathematical and Statistical Developments of Evolutionary Theory (S. Lessard, ed.). Proc. NATO ASI Symp., Kluwer, Dordrecht, 1990, 117228.

[28] R. C. Griffiths, On the distribution of points in a Poisson Dirichlet process, J. Appl. Probab. 25 (1988), 336-345.

[29] T. Hida and H. Nomoto, Gaussian measure on the projective limit space of spheres, Proc. Japan Academy, 40 (1964), 31-34.

[30] Ts. Ignatov, On a constant arising in the asymptotic theory of symmetric groups and on Poisson-Dirichlet measures, Teor. Veroyatnost. Primenen., 27 (1982), no. 1, 129-140 (Russian); English translation: Theory Probab. Appl. 27 (1982), 136-147.

[31] A. R. Its, A. G. Izergin, V. E. Korepin, N. A. Slavnov, Differential equations for quantum correlation functions, Intern. J. Mod. Phys., B4 (1990), 10031037.

[32] V. Ivanov and G. Olshanski, Kerov's central limit theorem for the Plancherel measure on Young diagrams, in: Symmetric Functions 2001: Surveys of Developments and Perspectives (S. Fomin, ed.). NATO Science Series II. Mathematics, Physics and Chemistry, vol. 74, Kluwer, 2001, 93151. 
[33] K. Johansson, Discrete orthogonal polynomial ensembles and the Plancherel measure, Ann. Math. (2), 153 (2001), no. 1, 259-296; math/9906120

[34] S. V. Kerov, Combinatorial examples in the theory of AF-algebras, in: Differential geometry, Lie groups and mechanics X, Zapiski Nauchnykh Seminarov LOMI 172 (1989), 55-67 (Russian); English translation: J. Soviet Math. 59 (1992), no. 5, 1063-1071.

[35] S. V. Kerov, Generalized Hall-Littlewood symmetric functions and orthogonal polynomials, in: Representation Theory and Dynamical Systems (A. M. Vershik, ed.), Advances in Soviet Math., Vol. 9, Amer. Math. Soc., Providence, R.I., 1992, 67-94.

[36] S. V. Kerov, Gaussian limit for the Plancherel measure of the symmetric group, Comptes Rendus Acad. Sci. Paris, Série I, 316 (1993), 303-308.

[37] S. V. Kerov, Subordinators and the actions of permutations with quasiinvariant measure, in: Zapiski Nauchnyh Seminarov POMI 223 (1995), 181-218 (Russian); English translation: J. Math. Sci.(New York) 87 (1997), no. $6,4094-4117$.

[38] S. V. Kerov, Anisotropic Young diagrams and Jack symmetric functions, Funktsional. Anal. Prilozhen. 34 (2000), no. 1, 51-64 (Russian); English translation: Funct. Anal. Appl., 34 (2000), no. 1, 41-51.

[39] S. Kerov, A. Okounkov and G. Olshanski, The boundary of Young graph with Jack edge multiplicities, Intern. Math. Res. Notices, 1998:4 (1998), 173-199; q-alg/9703037.

[40] S. Kerov and G. Olshanski, Polynomial functions on the set of Young diagrams, Comptes Rendus Acad. Sci. Paris Sér. I, 319 (1994), 121-126.

[41] S. Kerov, G. Olshanski and A. Vershik, Harmonic analysis on the infinite symmetric group. A deformation of the regular representation, Comptes Rendus Acad. Sci. Paris, Sér. I, 316 (1993), 773-778; detailed version in preparation.

[42] S. V. Kerov and N. V. Tsilevich, Stick breaking process generates virtual permutations with Ewens distribution, in: Zapiski Nauchnyh Seminarov POMI 223 (1995), 162-180 (Russian); English translation: J. Math. Sci. (New York), 87 (1997), no. 6, 4082-4093.

[43] J. F. C. Kingman, Random discrete distributions, J. Royal Statist. Soc. B, 37 (1975), 1-22.

[44] J. F. C. Kingman, The population structure associated with the Ewens sampling formula, Theoret. Population Biology, 11 (1977), 274-283.

[45] J. F. C. Kingman, Random partitions in population genetics, Proc. Roy. Soc. London A., 361 (1978), 1-20. 
[46] J. F. C. Kingman, The representation of partition structures, J. London Math. Soc. (2), 18 (1978), 374-380.

[47] J. F. C. Kingman, Poisson processes, Oxford University Press, 1993.

[48] A. Lenard, Correlation functions and the uniqueness of the state in classical statistical mechanics, Comm. Math. Phys, 30 (1973), 35-44.

[49] O. Macchi, The coincidence approach to stochastic point processes, Adv. Appl. Prob., 7 (1975), 83-122.

[50] O. Macchi, The fermion process - a model of stochastic point process with repulsive points, in: Transactions of the Seventh Prague Conference on Information Theory, Statistical Decision Functions, Random Processes and of the Eighth European Meeting of Statisticians (Tech. Univ. Prague, Prague, 1974), Vol. A, Reidel, Dordrecht, 1977, 391-398.

[51] F. J. Murray and J. von Neumann, On rings of operators IV, Ann. Math. 44 (1943), 716-808.

[52] M. L. Mehta, Random matrices, 2nd edition, Academic Press, 1991.

[53] Yu. A. Neretin, Hua type integrals over unitary groups and over projective limits of unitary groups, Duke Math. J., 114 (2002), 239-266; math-ph/0010014

[54] T. Nagao, M. Wadati, Correlation functions of random matrix ensembles related to classical orthogonal polynomials, J. Phys. Soc. Japan, 60 (1991), no. $10,3298-3322$.

[55] M. A. Naimark, Normed rings, Nauka, Moscow, 1962 (Russian); English translation: Normed algebras, Wolters-Noordhoff, Groningen, 1972.

[56] A. Yu. Okounkov, Thoma's theorem and representations of infinite bisymmetric group, Funktsion. Anal. Prilozhen. 28 (1994), no. 2, 31-40 (Russian); English translation: Funct. Anal. Appl., 28 (1994), no. 2, 101-107.

[57] A. Yu. Okounkov On representations of the infinite symmetric group, in: Representation Theory, Dynamical Systems, Combinatorial and Algorithmic Methods II, Zap. Nauchn. Semin. POMI (A. M. Vershik, ed.) 240 (1997), 167-229 (Russian); English translation: J. Math. Sci. (New York), 96 (1999), no. 5, 3550-3589.

[58] A. Okounkov, $S L(2)$ and $z$-measures, in: Random matrix models and their applications (P. M. Bleher and A. R. Its, eds). Mathematical Sciences Research Institute Publications 40, Cambridge Univ. Press, 2001, 407-420; math/0002136

[59] A. Okounkov, Infinite wedge and measures on partitions, Selecta Math. (New Ser.) 7 (2001), 57-81; math/9907127 
[60] A. Okounkov and G. Olshanski, Shifted Schur functions, Algebra i Analiz, 9 (1997), no. 2, 73-146 (Russian); English translation: St. Petersburg Math. J. 9 (1998), no. 2, 239-300.

[61] G. Olshanski, Unitary representations of infinite-dimensional pairs $(G, K)$ and the formalism of $R$. Howe, Doklady AN SSSR, 269 (1983), 33-36 (Russian); English translation: Soviet Math. Doklady, 27 (1983), no. 2, 290-294.

[62] G. Olshanski, Unitary representations of infinite-dimensional pairs $(G, K)$ and the formalism of $R$. Howe, in: Representation of Lie Groups and Related Topics (A. Vershik and D. Zhelobenko, eds.), Advanced Studies in Contemporary Math. 7, Gordon and Breach Science Publishers, New York etc., 1990, 269-463.

[63] G. Olshanski, Unitary representations of $(G, K)$-pairs connected with the infinite symmetric group $S(\infty)$, Algebra i Analiz, 1 (1989), no. 4, 178-209 (Russian); English translation: Leningrad Math. J. 1 (1990), 983-1014.

[64] G. Olshanski, The problem of harmonic analysis on the infinitedimensional unitary group, J. Funct. Anal., to appear; math/0109193

[65] G. Olshanski, A. Regev and A. Vershik, Frobenius-Schur functions, in: Studies in Memory of Issai Schur (A. Joseph, A. Melnikov, R. Rentschler, eds.), Birkhäuser, to appear; math/0110077.

[66] G. Olshanski, Point processes and the infinite symmetric group. Part I: The general formalism and the density function, 1998, math/9804086

[67] A. Borodin, Point processes and the infinite symmetric group. Part II: Higher correlation functions, 1998, math/9804087.

[68] A. Borodin and G. Olshanski, Point processes and the infinite symmetric group. Part III: Fermion point processes, 1998, math/9804088.

[69] A. Borodin, Point processes and the infinite symmetric group. Part IV: Matrix Whittaker kernel, 1998, math/9810013.

[70] G. Olshanski, Point processes and the infinite symmetric group. Part V: Analysis of the matrix Whittaker kernel, 1998, math/9810014.

[71] D. Pickrell, Measures on infinite dimensional Grassmann manifold, J. Funct. Anal., 70 (1987), 323-356.

[72] N. A. Rozhkovskaya, Multiplicative distributions on Young graph, in: Representation Theory, Dynamical Systems, Combinatorial and Algorithmic Methods II (A. M. Vershik, ed.), Zapiski Nauchnykh Seminarov POMI 240, Nauka, St. Petersburg, 1997, 246-257 (Russian); English translation: J. Math. Sci. (New York) 96 (1999), no. 5, 3600-3608 
[73] H. Shimomura, On the construction of invariant measure over the orthogonal group on the Hilbert space by the method of Cayley transformation, Publ. RIMS Kyoto Univ., 10 (1974/75), 413-424.

[74] A. Soshnikov, Determinantal random point fields, Uspekhi Mat. Nauk 55 (2000), no. 5, 107-160 (Russian); English translation: Russian Math. Surveys, 55 (2000), no. 5, 923-975; math/0002099.

[75] S. Stratila and D. Voiculescu, Representations of AF-algebras and of the group $U(\infty)$, Springer Lecture Notes 486, 1975.

[76] E. Thoma, Die unzerlegbaren, positive-definiten Klassenfunktionen der abzählbar unendlichen, symmetrischen Gruppe, Math. Zeitschr., 85 (1964), 40-61.

[77] E. Thoma, Characters of infinite groups, in: Operator Algebras and Group Representations (Gr. Arsene, S. Strătilă, A. Verona, and D. Voiculescu, eds), Vol. 2, Pitman, 1984, pp. 23-32.

[78] C. A. Tracy and H. Widom, Universality of distribution functions of random matrix theory II, in: Integrable Systems: From Classical to Quantum (J. Harnad, G. Sabidussi, and P. Winternitz, eds). CRM Proceedings \& Lecture Notes 26. Amer. Math. Soc., Providence, 2000, pp. 251-264.

[79] A. M. Vershik, Description of invariant measures for the actions of some infinite-dimensional groups, Doklady AN SSSR, 218 (1974), 749-752 (Russian); English translation: Soviet Math. Doklady, 15 (1974), 1396-1400.

[80] A. M. Vershik, Asymptotic distribution of decompositions of natural numbers into prime divisors, Dokl. Akad. Nauk SSSR, 289 (1986), no. 2, 269272; English translation: Soviet Math. Doklady, 34 (1987), 57-61.

[81] A. M. Vershik, Statistical mechanics of combinatorial partitions, and their limit shapes, Funktsional. Anal. Prilozhen. 30 (1996), no. 2, 19-39 (Russian); English translation: Funct. Anal. Appl., 30 (1996), 90-105.

[82] A. M. Vershik and S. V. Kerov, Characters and factor representations of the infinite symmetric group, Doklady AN SSSR, 257 (1981), 1037-1040 (Russian); English translation: Soviet Math. Doklady 23 (1981), 389-392.

[83] A. M. Vershik and S. V. Kerov, Asymptotic theory of characters of the symmetric group, Funktsion. Anal. Prilozhen., 15 (1981), no. 4, 15-27 (Russian); English translation: Funct. Anal. Appl., 15 (1981), no. 4, 246-255.

[84] A. M. Vershik and S. V. Kerov, Characters and factor representations of the infinite unitary group, Doklady AN SSSR, 267 (1982), no. 2, 272-276 (Russian); English translation: Soviet Math. Doklady, 26 (1982), 570-574. 
[85] A. M. Vershik and S. V. Kerov, Locally semisimple algebras. Combinatorial theory and the $K_{0}$ functor, in: Itogi Nauki, Sovr. Probl. Mat., Noveish. Dostizh., VINITI, 26 (1985), 3-56 (Russian); English translation: J. Soviet Math., 38 (1987), 1701-1733.

[86] A. M. Vershik and A. A. Shmidt, Limit measures arising in the asymptotic theory of symmetric groups I, II, Teor. Veroyatnost. Primenen., 22 (1977), no. 1, 72-88, 23 (1978), no. 1, 42-54 (Russian); English translation: Theory of Probab. Appl. 22 (1977), 70-85, 23 (1978), 36-49.

[87] D. Voiculescu, Représentations factorielles de type $\mathrm{II}_{1}$ de $U(\infty)$, J. Math. Pures Appl., 55 (1976), 1-20 .

[88] A. J. Wassermann, Automorphic actions of compact groups on operator algebras, Thesis, University of Pennsylvania, 1981.

[89] G. A. Watterson, The stationary distribution of the infinitely many-alleles diffusion model, J. Appl. Probab., 13 (1976), 639-651.

[90] Y. Yamasaki, Projective limit of Haar measures on $O(n)$, Publ. RIMS, Kyoto Univ., 8 (1972/73), 141-149.

[91] Y. Yamasaki, Kolmogorov's extension theorem for infinite measure, Publ. RIMS, Kyoto Univ., 10 (1974/75), 381-411. 